\title{
Journal of Advanced Materials Science and Engineering
}

\section{Electrospray Deposition of Multiwalled Carbon Nanotubes-OH/ Polyvinylidene Fluoride Nanocomposites for Volatile Organic Compounds (VOCs) Detection}

\author{
Akinwunmi Joaquim, Omari Paul, Anthony Thai, Aya Jones, April Falconer, Richard Mu* and \\ Frances Williams*
}

College of Engineering, Tennessee State University, Nashville TN, 37209, USA.
"Correspondence:

Richard $\mathrm{Mu}$ and Frances Williams, College of Engineering, Tennessee State University, Nashville TN, 37209, USA.

Received: 19 Septemnber 2021; Accepted: 15 October 2021

Citation: Akinwunmi J, Omari P, Anthony T, et al. Electrospray Deposition of Multiwalled Carbon Nanotubes-OH/ Polyvinylidene Fluoride Nanocomposites for Volatile Organic Compounds (VOCs) Detection. J Adv Mater Sci Eng. 2021; 1(1): 1-6.

\begin{abstract}
In this work, we report on the synthesis and performance evaluation of electrosprayed multi-walled carbon nanotubes (MWCNTS)/ Polyvinylidene Fluoride (PVDF) nanocomposite materials for gas detection of volatile organic compounds. MWCNTs are characterized by a hollow structure, large surface area and several unique properties that make them potentially excellent for gas sensing.

However, in order to improve their sensitivity, stability and reproducibility effects, the carbon nanotubes were doped with PVDF polymer in a minimal concentration such that it utilizes its strong binding effect with interconnected nanotubes and the substrate and further, the polymer does not affect the dominance of MWCNTs on the surface as observed from SEM characterization. The electrospray deposition technique, combined with sonication, was used to ensure the MWCNT was dispersed evenly in the polymer matrix, as MWCNTs tend to aggregate due to their strong Van der Waals forces.

The sensing mechanism and the kinetic adsorption of gases on the sensors were studied and the experimental result shows that MWCNT-OH/PVDF sensor revealed a larger sensitivity than that of MWCNT-OH sensor to tested VOCs at room temperature.
\end{abstract}

\section{Keywords}

Electrospray deposition, Multiwalled carbon nanotubes, Polyvinylidene Fluoride, Sensors.

\section{Introduction}

Carbon based nanomaterial Carbon Nanotubes (CNTs) has gained a lot of interest among researchers and scientist after its discovery by Ijinma in 1991 [1]. They possess outstanding electrical, thermal, mechanical and chemical properties due to their structure and have shown great promise as active materials in sensor and micro-electromechanical systems (MEMS) technology [2]. Studies have been carried out on the use of CNTs importantly as gas sensors $[3,4]$. It was found out that the large surface area to volume ratio provided by the hollow structure of the nanotubes and their inside walls provided large adsorptive capacity for gas detection. However, during gas detection using pristine CNTs, potential shortcomings could arise which includes lack of specificity to various gaseous analytes and low sensitivity towards analytes that possess little or no affinity to CNTs. These drawbacks have been studied and shown that functionalization of the CNTs surface with analyte specific entities has an effect on the binding energy and therefore improving the dynamic response of the CNT based sensors [5]. Also, CNTs tend to aggregate due to their strong vanderwall interactions, therefore modification of their surfaces with oxygen rich chemical groups such as carboxyl, hydroxyl, and carbonyl could promote dispersion and biocompatibility when 
incorporated with materials such as polymers [6]. Among several polymers used in CNT/polymer nanocomposites is polyvinylidene fluoride (PVDF). PVDF is a well-known lightweight, flexible, mechanically stable and non-toxic piezoelectric polymer that offers good physical and chemical properties [7]. It is worthy to note that as a chemical sensor, PVDF has showed good response to dimethyl methylphosphonate (DMMP), a stimulant of nerve agents but when tested to traditional volatile organic compounds such as acetone and ethanol its sensor response was really poor [8]. However, taking advantage of its biocompatibility and binder properties, the PVDF incorporated with the CNTs could lead to uniform film fabrication and sufficient mechanical adhesion to the sensor surface [9]. Amongst several sensors used for gas detection [10], QCM sensor has been chosen in this work because it is simple to operate, highly sensitive and can detect target gaseous analytes at room temperature. QCM sensors operate on the principle of the piezoelectric effect of its quartz crystal and can determine even nanogram levels of mass changes. With several methods used to coat a sensing layer onto the surface of a QCM electrode such as spin coating, drop casting, electro spraying or electro spinning with each methods having their various advantages and disadvantages its therefore important to state that the right choice of deposition methods and appropriate sensing coating has a great influence in making the QCM sensors more sensitive or selective [9].

In this work, electrospray deposition technique has been used to create MWCNT-OH and MWCNT-OH/PVDF based QCM sensors for detection of volatile organic compounds. The electrospray deposition technique chosen favors very good dispersion of the material on the QCM surface due to coulomb repulsive forces which would consequently aid better gas adsorption. We also report on the kinetic adsorption studies of ethanol and acetone vapor on the MWCNT-OH and MWCNT-OH/PVDF gas sensors using the pseudo first order nonlinear equations. The coefficient of determination $\left(\mathrm{R}^{2}\right)$ was used to determine the best fitting equation and other rate kinetic parameters were determined.

\section{Experimental}

\section{Chemicals}

PVDF (average molecular weight $\sim 534,000$ ), MWCNT$\mathrm{OH}$ (diameter: 10-20nm, length: $10-30 \mu \mathrm{m}$ ) and $\mathrm{N}$, N-Dimethylformamide (DMF) used to prepare the sensitive coatings were purchased from Sigma-Aldrich. Ethanol and Acetone (99.5\%) used as target analytes were purchased from VWR chemicals. Ultra-high purity nitrogen was used as the carrier and purge gas. All chemicals were used without any further purification.

\section{Material Synthesis}

The material synthesis part involved creating of two different suspensions. MWCNT-OH powder was suspended in DMF solvent and sonicated using a water bath sonicator to achieve a homogenous suspension. The MWCNT-OH suspension is made at $1 \%$ by weight in solvent. The other suspension involves the mixture of $1 \%$ by weight of PVDF in DMF with $1 \%$ by weight of MWCNT-OH in DMF; this mixture was sonicated using a water bath sonicator for 4 hours to prevent aggregation of the MWCNT$\mathrm{OH}$ in the PVDF polymer. This suspension resulted in MWCNT$\mathrm{OH} / \mathrm{PVDF}$ in ratio 1:1.

\section{Fabrication of MWCNT-OH and MWCNT-OH/PVDF Based QCM Gas Sensors}

AT cut QCM crystals, and its oscillator circuit were purchased from Instruments for intelligent control (INFICON) company. The Quartz crystal which had a diameter of $14 \mathrm{~mm}$ was rinsed with ethanol before being used. For deposition, the QCM crystals and silicon wafer were placed in the electrospray deposition system. Electrospray uses a syringe and pump to control the rate at which the suspension is delivered through a highly charged needle. The charge on the needle is supplied by a high voltage power source. The parameters within the electrospray system were optimized to ensure good spray and film formation. The samples were deposited onto the substrate at a height of $12 \mathrm{~cm}$ with voltages applied between $13-15 \mathrm{KV}$, solution flow rate of $0.3 \mathrm{ml} / \mathrm{hr}$. and deposition time of $15 \mathrm{mins}$. After deposition, the crystal and wafers were kept in an oven at $50^{\circ} \mathrm{C}$ for 2 hours to ensure complete evaporation of solvent and to promote film stability. The frequency shift caused by film deposition was calculated by subtracting the resonance frequency before coating from the frequency after the film had been dried. The frequency change of coating the MWCNT-OH was about $190.7 \mathrm{~Hz}$ and MWCNT-OH/PVDF was $204.2 \mathrm{~Hz}$. The morphology of the films was analyzed using a SEM.

\section{Experimental setup}

To investigate the sensitive nature of MWCNT-OH and MWCNTOH/PVDF based QCM sensors, a characterization system was developed in our lab. The experiments were carried out in a sealed chamber with 13.7 liter in volume at room temperature. The resonance frequency shifts of the QCM sensors due to gas adsorption were measured using a (MAXTEK thickness monitor model TM-400). Nitrogen was first purged into the chamber to eliminate any form of moisture and to ensure that frequency changes are only due to adsorption of tested VOCs. Once a stable frequency line was observed, the VOC was introduced into the chamber using the nitrogen bubbling method. After the sample vapor was dispersed in the chamber, frequency shifts began to occur and got to the point of saturation in few minutes. After the frequency reached equilibrium, Nitrogen was purged again in a desorption process to remove the adsorbed analyte molecules until the frequency returned to its initial value, this reversal process made it possible for the sensor to be ready for its next measurement.

\section{Results and Discussion \\ Morphology of the Coated Films}

The morphology of the MWCNT-OH and MWCNT-OH/PVDF has been characterized using a SEM, as reported in Figures 1(a) and 1(b). The materials were deposited on a silicon substrate for electron microscopy observations. Both samples show good surface coverage. The polymer inclusion doesn't affect the dominance of the nanotubes in the MWCNT-OH/PVDF sample as shown in the Figure 1(b), although there's a change in the color intensity indicating the presence of the PVDF and as observed 

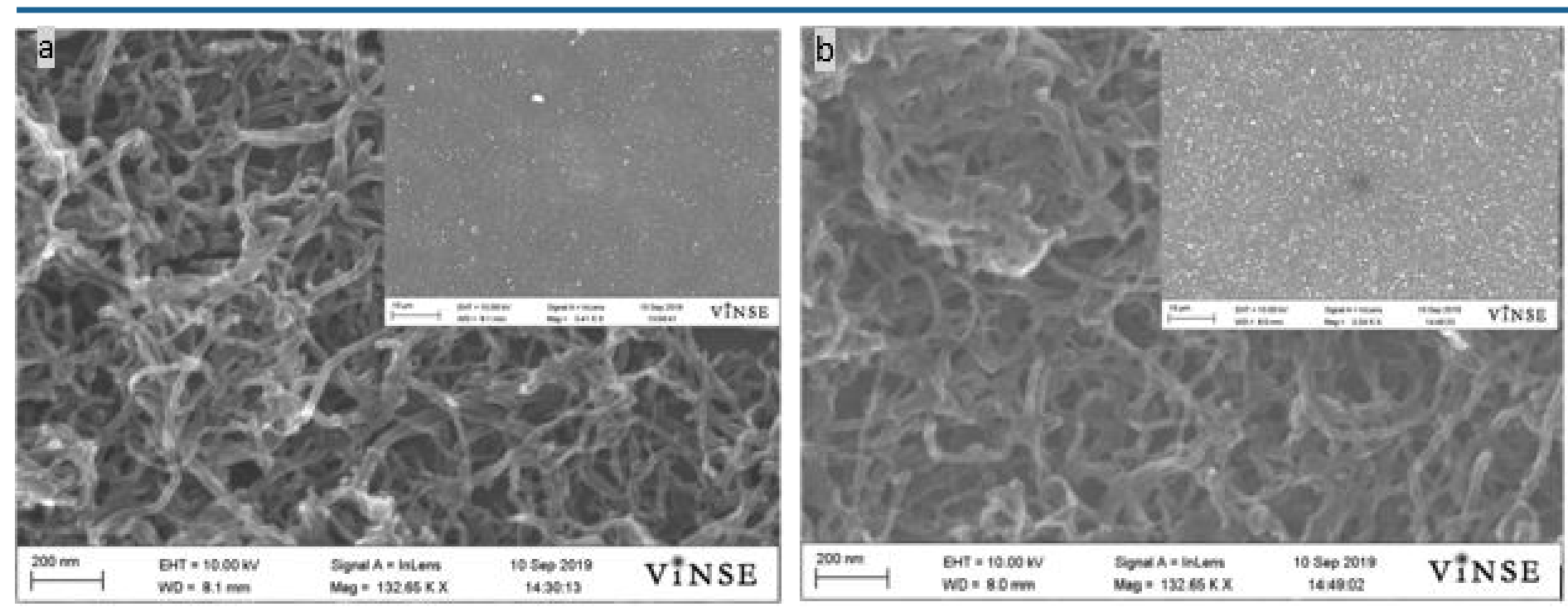

Figure 1: SEM images of (a) MWCNT-OH film and (b) MWCNT-OH/PVDF nanocomposite film.

from the MWCNT-OH/PVDF inset a lot of bright white spots which could be as a result of PVDF not being able to discharge the electrons accumulated on the sample surface or otherwise known as the charging effect.

\section{Response of Sensors to VOCs}

It was observed from our experimental measurements that each tested VOCs has its own impact on the frequency change, which is important for the detection and identification of gases. Measured results for MWCNT-OH sensor to ethanol and acetone vapors are presented in Figures 2 (a) and (b). Ethanol showed a higher increase in frequency change compared to acetone, and while comparing the reference QCM to MWCNT-OH sensor in the detection of ethanol, the MWCNT-OH was about $13.8 \mathrm{~Hz}$. This value is $\sim 3.5$ times higher than the frequency shift of the reference QCM.

The highest mass sensitivity has been achieved for MWCNT-OH/ PVDF sensor due to the greatest gas adsorbed mass as shown in Figures 2 (c) and (d). The MWCNT-OH/PVDF sensor in comparison to the reference QCM in the detection of ethanol performed better having a frequency change of $24.1 \mathrm{~Hz}$. This value being $\sim 6.5$ times higher than the frequency shift of the reference QCM. The repeatability of the reference, MWCNT-OH and MWCNT-OH/PVDF QCM sensors were also carried out by exposing the sensors to VOCs in three different test cycles as presented in Figures 3(a) and 3(b). We observed very good repeatability of the sensors with little variations which could be due to incomplete desorption of tested VOC.

\section{Sensing Mechanism}

In general, the gas adsorption can be considered as the contribution of the main sensing mechanism of mass loading for the MWCNT$\mathrm{OH}$ and MWCNT-OH/PVDF sensors. When both sensors are exposed to VOC molecules, the frequency decreases rapidly with time as a result of surface adsorption effect. Then, the rate of the frequency decrease begins to slow down due to bulk diffusion effect. After getting to a point of saturation, the frequency attains a stable value.
The magnitude of the MWCNT-OH sensor to ethanol and acetone could be attributed to the physical adsorption of their vapors on the MWCNT-OH surfaces via Van Der Waals forces which is in good agreement with previous work [10]. Since ethanol is a polar molecule due to the presence of $-\mathrm{OH}$ group, it can form strong hydrogen bonds with the sensing material; as a result of this, more molecules were adsorbed and provided higher frequency shift, however acetone is a non-polar molecule and there is no hydrogen bond formation. The MWCNT-OH/PVDF sensor adsorbed the greater amount of the ethanol and acetone vapor compared to MWCNT-OH sensor which could be due to increase in the surface area of the nanotubes as a result of the PVDF polymer filling the inner structure of the MWCNT-OH as previously reported in this work [11] and also the interaction between the PVDF polymer and the OH-functionalized nanotubes via hydrogen bonding could promote debundling of the tubes, consequently leading to more gas adsorption.

\section{Theoretical Studies and Model Fitting}

Kinetic studies of adsorption unto the sensitive coatings deposited on the QCM sensors are necessary to evaluate the effectiveness of an adsorption process. It is well known that the adsorption rate and the equilibrium adsorption amount are determined by both the gas molecules and sensor materials. By this study we would be able to obtain a conceptual understanding of the adsorption mechanism associated with the phenomena [12]. The kinetics of the gas adsorption process is based on the fitting of mass-time curves. The exponential fitting used in the figures corresponds to a first order non-linear isotherm as shown in Equation (1).

$M_{t}=M_{e}\left(1-e^{-k t}\right)$

Where $M_{t}$ is the adsorbed mass at time $t, M_{e}$ is the equilibrium adsorption amount obtained from the fitting of experimental data, $k$ is the adsorption factor and $t$ is the time.

Since we already have the frequency shift results gotten from the experiments, to obtain the adsorbed mass $\left(M_{t}\right)$, the sauerbrey equation was employed as shown in Equation (2) 

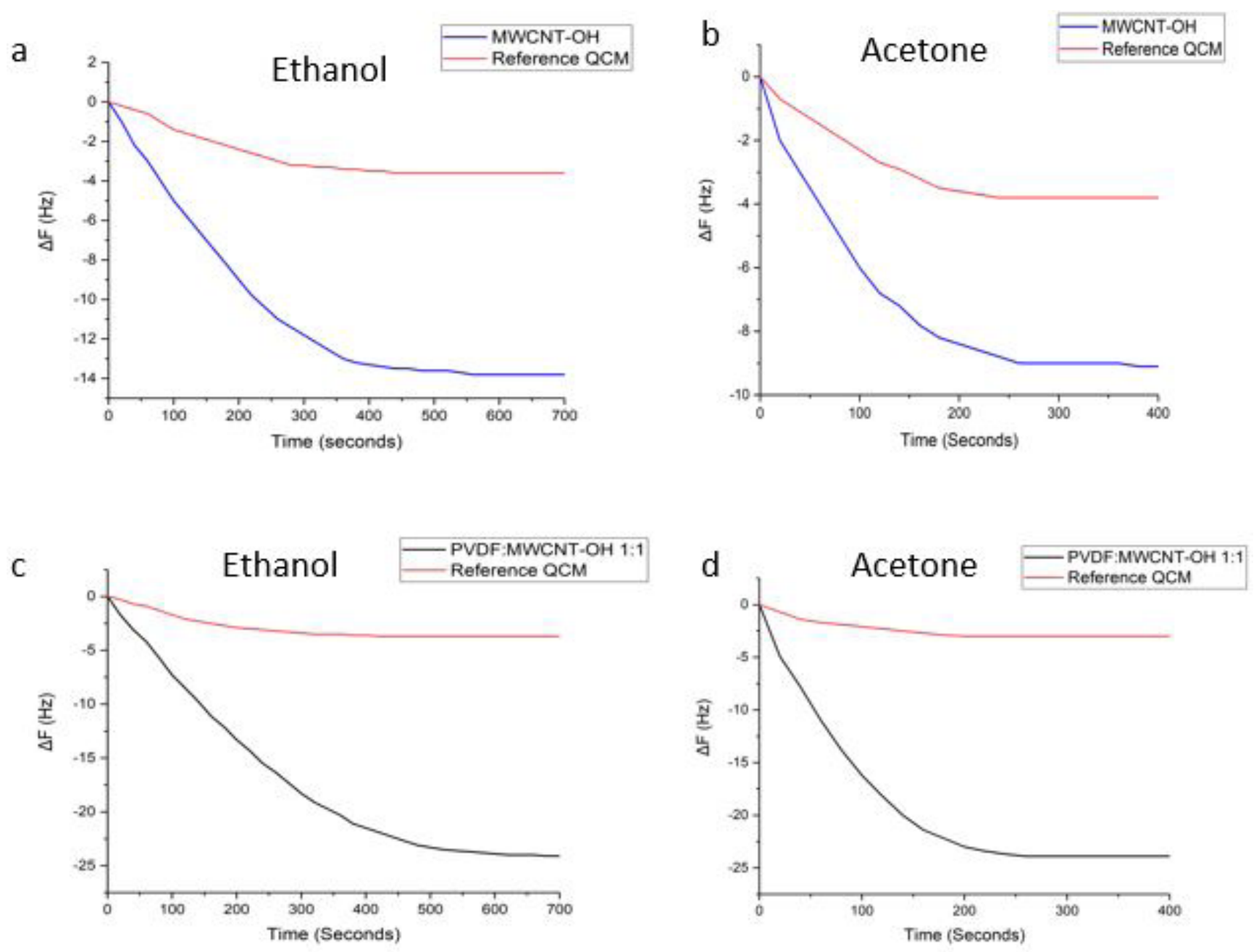

Figure 2: Reference vs. MWCNT-OH sensor response to vapors of (a) ethanol and (b) acetone and Reference vs. MWCNT-OH/PVDF sensor response to vapors of (c) ethanol and (d) acetone.
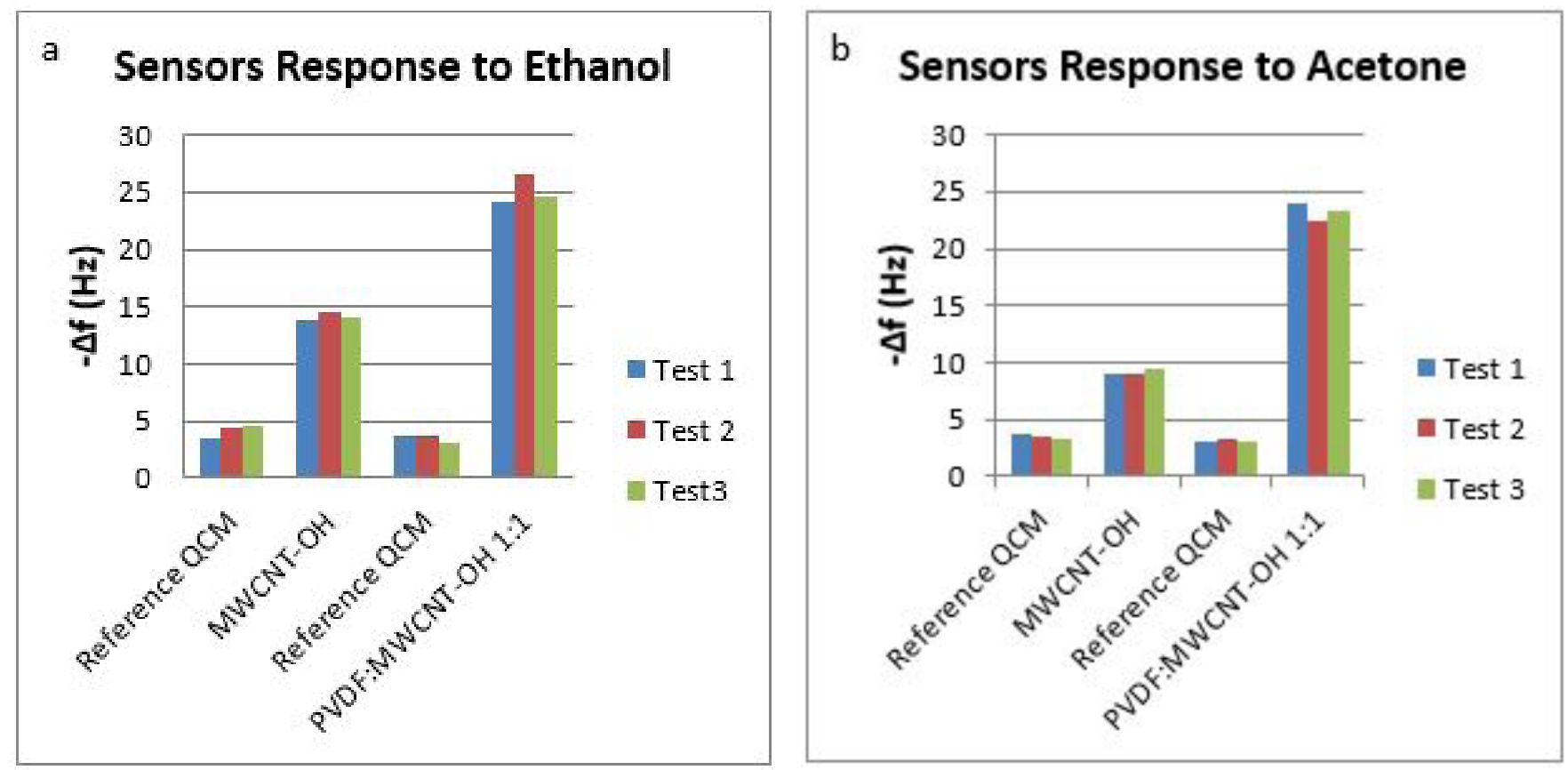

Figure 3: Repeatability of Reference, MWCNT-OH and MWCNT-OH/PVDF sensor to (a) Ethanol and (b) Acetone. 

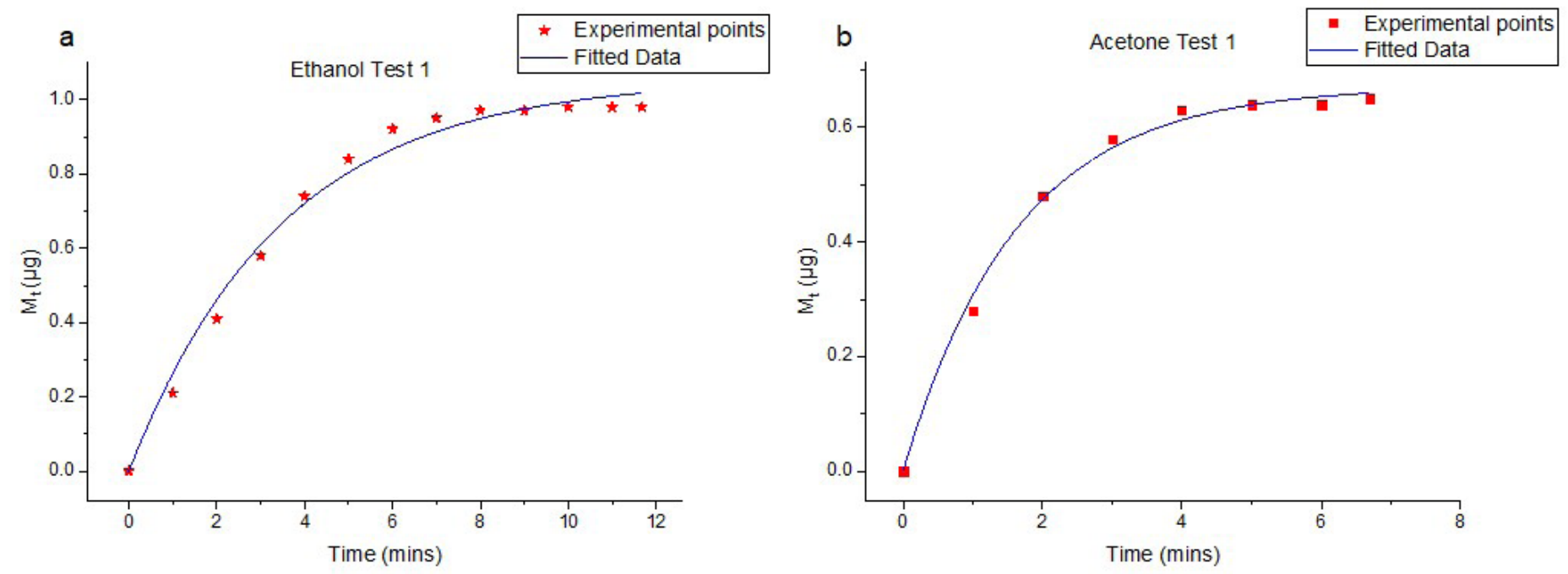

Figure 4: MWCNT-OH sensor adsorption and nonlinear fitting to (a) Ethanol and (b) Acetone.
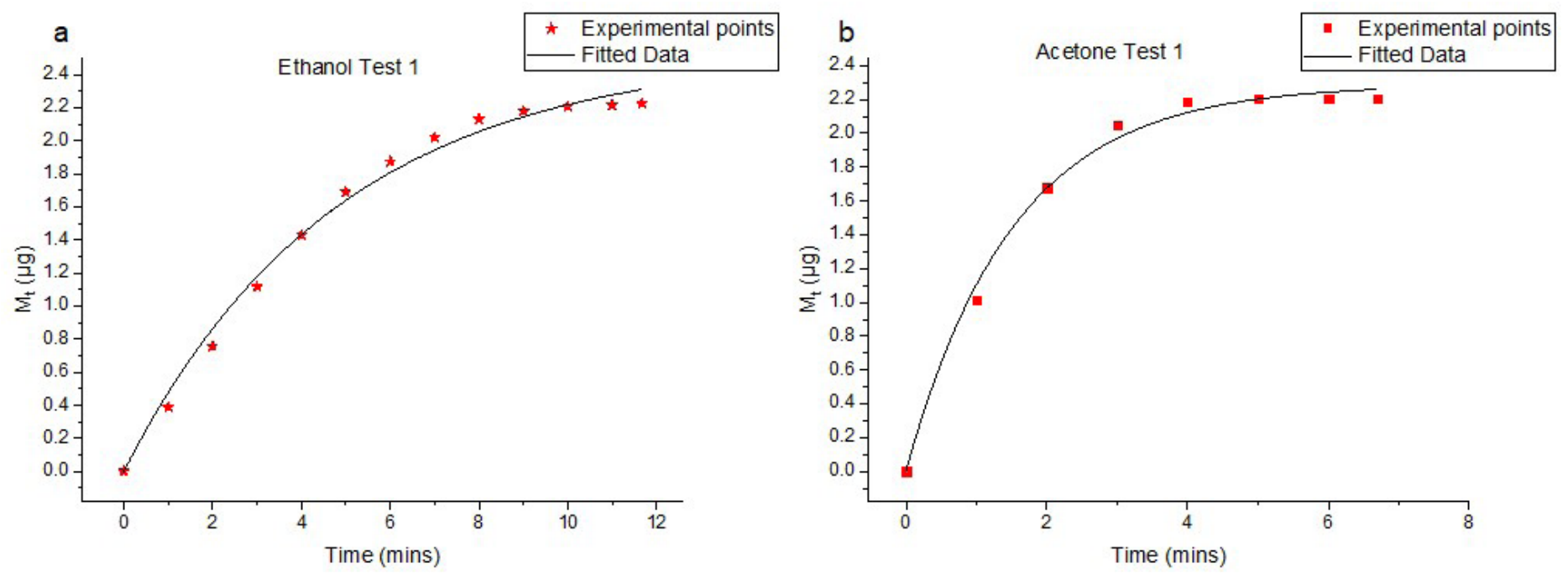

Figure 5: MWCNT-OH/PVDF sensor adsorption and nonlinear fitting to (a) Ethanol and (b) Acetone.

$\Delta f=-2.26 \times 10^{-6} f_{0}^{2}\left(\frac{\Delta m}{A}\right)$

Where $\Delta f$ is the change in frequency and $f_{0}$ is the resonance frequency of QCM crystals coated with the sensitive materials both measured in $\mathrm{Hz}, \mathrm{A}$ is the active surface area of deposited materials in $\mathrm{cm}^{2}$ and $\Delta m \mathrm{~s}$ the calculated mass loading in $\mathrm{g}$.

The nonlinear isotherm as shown in Equation (1) is used as an approximation for nonlinear systems with pore or surface adsorption and diffusion mechanism. The main advantage of this approximation is in its simplistic formula to describe a no reaction case and thus it can be used to model a physisorption reaction [13].

Therefore, this kinetic study was performed to understand the adsorption mechanism of acetone and ethanol vapor on MWCNT$\mathrm{OH}$ and MWCNT-OH/PVDF coated QCM sensors at room temperature. The nonlinear plots for the first cycle test of $M_{t}$ vs. $t$ were plotted in figures 4 and 5 for both sensors response to target vapors, this yielded the first order rate constants $k$ and $M_{e}$ values. The $M_{e}$ values calculated from fitting of experimental data with nonlinear pseudo first order kinetics are shown in Table 1 for MWCNT-OH and MWCNT-OH/PVDF in Table 2. The recorded data reveals a faster kinetics for the adsorption of acetone vapor on both sensors compared to ethanol vapor in which most of the adsorption occurs in the early minutes of adsorption experiments and then both sensors got to the point of saturation. We observe slight variations in the $k$ values in repeated adsorption tests of the vapors to the sensors; this could be due to minor fluctuations in the pressure of the nitrogen carrier gas while using the bubbling method to convey the vapor into the chamber, the work done by Maedeh Delavar et al. justifies this assumption [14].

\section{Conclusions}

MWCNT-OH and MWCNT-OH/PVDF coated QCM sensors have been prepared by electrospray deposition for enhanced mass sensitivity to detect VOCs of ethanol and acetone at room 
Table 1: Kinetic adsorption of Acetone and Ethanol VOCs unto MWCNT-OH sensor.

\begin{tabular}{|c|c|c|c|c|c|}
\hline \multirow{2}{*}{ Adsorbent } & \multirow{2}{*}{ VOC } & \multirow{2}{*}{$\mathrm{Mt}_{\mathrm{exp}}[\boldsymbol{\mu g}]$} & \multicolumn{3}{|c|}{ Non- Linear Pseudo First order } \\
\hline & & & Me $[\mu \mathrm{g}]$ & $\mathbf{K}$ & $\mathbf{R}^{2}$ \\
\hline \multirow{6}{*}{ MWCNT-OH } & Acetone-Test 1 & 0.65 & 0.67 & 0.61 & 0.995 \\
\hline & Acetone-Test 2 & 0.63 & 0.66 & 0.61 & 0.997 \\
\hline & Acetone- Test 3 & 0.67 & 0.69 & 0.73 & 0.997 \\
\hline & Ethanol- Test 1 & 0.98 & 1.05 & 0.29 & 0.988 \\
\hline & Ethanol- Test 2 & 1.04 & 1.10 & 0.34 & 0.986 \\
\hline & Ethanol- Test 3 & 1.02 & 1.08 & 0.28 & 0.991 \\
\hline
\end{tabular}

Table 2: Kinetic adsorption of Acetone and Ethanol VOCs unto MWCNT-OH/PVDF sensor.

\begin{tabular}{|c|c|c|c|c|c|}
\hline \multirow{2}{*}{ Adsorbent } & \multirow{2}{*}{ VOC } & \multirow{2}{*}{$\mathbf{M t}_{\text {exp }}[\mu \mathrm{g}]$} & \multicolumn{3}{|c|}{ Non- Linear Pseudo First order } \\
\hline & & & Me $[\mu \mathrm{g}]$ & $\mathbf{K}$ & $\mathbf{R}^{2}$ \\
\hline \multirow{6}{*}{ MWCNT-OH/PVDF } & Acetone-Test 1 & 2.20 & 2.29 & 0.66 & 0.995 \\
\hline & Acetone-Test 2 & 2.07 & 2.22 & 0.47 & 0.995 \\
\hline & Acetone- Test3 & 2.16 & 2.26 & 0.59 & 0.993 \\
\hline & Ethanol- Test 1 & 2.22 & 2.53 & 0.21 & 0.992 \\
\hline & Ethanol- Test 2 & 2.46 & 3.21 & 0.14 & 0.991 \\
\hline & Ethanol- Test 3 & 2.27 & 2.59 & 0.23 & 0.981 \\
\hline
\end{tabular}

temperature, and its response was compared to reference QCM sensor. The sensing response and repeatability of both coated sensors were investigated, the results revealed that the MWCNT$\mathrm{OH} / \mathrm{PVDF}$ coated sensor had highest sensitivity to ethanol vapor and this behavior was explained due to increase in surface area of the nanotubes. Good repeatability was obtained for both coated sensors to all analytes. In addition, kinetic adsorption studies were performed on both coated sensors, the results reveal a faster reaction mechanism for acetone compared to ethanol vapor and our experimental data best fit the pseudo first order nonlinear model for both acetone and ethanol adsorption indicating that the reaction is more inclined towards physisorption. Furthermore, due to room temperature operations, the MWCNT-OH and MWCNT-OH/ PVDF based QCM sensors show great promise for chemical detection and applications in industrial and environmental air monitoring.

\section{Acknowledgments}

This study was supported in parts by NSF HBCU-RISE Award \#: HRD-1924241; NSF-PREM Award \# 2122169; And a Lockheed Martin Corporation grant.

\section{References}

1. Iijima S. Helical microtubules of graphitic carbon. Nature. 1991; 354: 56-58.

2. Xie G, Xie T, Zhu T, et al. Fabrication of a gas sensor array with micro-wells for VOCs gas sensing based on polymer/ carbon nanotube thin films. In 7th International Symposium on Advanced Optical Manufacturing and Testing Technologies: Smart Structures and Materials for Manufacturing and Testing. 2014.

3. Zhao J, Buldum A, Han J, et al. Gas molecule adsorption in carbon nanotubes and nanotube bundles. Nanotechnology. 2002; 13: 195.

4. Li J, Lu Y, Ye Q, et al. Carbon nanotube sensors for gas and organic vapor detection. Nano letters. 2003; 3: 929-933.

5. Sun $\mathrm{P}$, Jiang $\mathrm{Y}$, Xie G, et al. Gas sensitivities of solvent functionalized CNTs to volatile organic compounds. In2009 International Conference on Apperceiving Computing and Intelligence Analysis 2009; 162-165.

6. Vardharajula S, Ali SZ, Tiwari PM, et al. Functionalized carbon nanotubes: biomedical applications. International journal of Nano medicine. 2012; 7: 5361.

7. Barnakov YA, Paul O, Joaquim A, et al. Light intensity-induced phase transitions in graphene oxide doped polyvinylidene fluoride. Optical Materials Express. 2018; 8: 2579-2585.

8. Ying Z, Jiang Y, Du X, et al. PVDF coated quartz crystal microbalance sensor for DMMP vapor detection. Sensors and Actuators B: Chemical. 2007; 125: 167-172.

9. Haghighi E, Zeinali S. Nanoporous MIL-101 (Cr) as a sensing layer coated on a quartz crystal microbalance (QCM) nanosensor to detect volatile organic compounds (VOCs). RSC advances. 2019; 9: 24460-24470.

10. Zhou Y, Jiang Y, Xie G, et al. Gas sensors based on multiplewalled carbon nanotubes-polyethylene oxide films for toluene vapor detection. Sensors and Actuators B: Chemical. 2014; 191: 24-30.

11. Birch ME, Ruda-Eberenz TA, Chai M, et al. Properties that influence the specific surface areas of carbon nanotubes and nanofibers. Annals of occupational hygiene. 2013; 57: 1148-1166.

12. Sohn S, Kim D. Modification of Langmuir isotherm in solution systems-definition and utilization of concentration dependent factor. Chemosphere. 2005; 58: 115-123.

13. Skodras G, Diamantopoulou I, Pantoleontos G, et al. Kinetic studies of elemental mercury adsorption in activated carbon fixed bed reactor. Journal of hazardous materials. 2008; 158: 1-3.

14. Delavar M, Ghoreyshi AA, Jahanshahi M, et al. Equilibria and kinetics of natural gas adsorption on multi-walled carbon nanotube material. RSC advances. 2012; 2: 4490-4497.

(C) 2021 Akinwunmi J, et al. This article is distributed under the terms of the Creative Commons Attribution 4.0 International License 